\title{
Dark Majorana particles from the minimal walking technicolor theory
}

\author{
Chris Kouvaris* \\ The Niels Bohr Institute, Blegdamsvej 17, DK-2100 Copenhagen Ø, Denmark, \\ University of Southern Denmark, Campusvej 55, DK-5230 Odense M, Denmark \\ and CERN Theory Division, CH-1211 Geneva 23, Switzerland
}

(Received 2 April 2007; published 18 July 2007)

\begin{abstract}
We investigate the possibility of a dark matter candidate emerging from a minimal walking technicolor theory. In this case, techniquarks as well as technigluons transform under the adjoint representation of $\mathrm{SU}(2)$ of technicolor. It is therefore possible to have technicolor neutral bound states between a techniquark and a technigluon. We investigate this scenario by assuming that such a particle can have a Majorana mass and we calculate the relic density. We identify the parameter space where such an object can account for the full dark matter density avoiding constraints imposed by the CDMS and the LEP experiments.
\end{abstract}

DOI: 10.1103/PhysRevD.76.015011

PACS numbers: $12.60 . \mathrm{Nz}, 95.35 .+\mathrm{d}$

\section{INTRODUCTION}

One of the most important open problems in modern physics is that of the origin of dark matter. In 1933, Zwicky realized that the mass from the bright part of the Coma cluster cannot explain the motion of galaxies at the edge of the cluster. He assumed that there must be some kind of mass, that does not interact "much" and therefore appears dark to us, that has to be present in order to explain the motion of the galaxies without changing the gravitational law. Since then, the origin of dark matter remains an enigma. There are two basic types of candidates for dark matter. In the first one belong objects usually referred as MACHOs (massive compact halo objects), mostly of baryonic origin. Objects like black holes, brown dwarf stars, and giant planets can be legitimate MACHO candidates. However, reliable observations have concluded that MACHOs cannot account for more than $20 \%$ of dark matter [1].

In the second type of candidates belong particles usually referred as WIMPs (weak interacting massive particles). These particles are usually of nonbaryonic origin and in principle can account for the whole dark matter density. There are some basic requirements that these particles have to fulfill. First of all, they have to be electrically neutral, since in order to be part of dark matter they should not couple to electromagnetism. In addition, WIMPs should be relatively heavy and therefore nonrelativistic, in order to be part of cold dark matter. Very light particles (as neutrinos, for example) would form hot dark matter. The existence of hot dark matter is not consistent with observations because the relativistic velocities of the particles smear out structure on small scales before the relic hot gas of light particles becomes nonrelativistic.

There are several dark matter candidates such as axions, supersymmetric particles, and technibaryons. There are also interesting alternative possibilities in literature [2-

*kouvaris@nbi.dk
5]. Dark matter candidates are constrained theoretically as well as experimentally. Several observations like those of WMAP give a rather precise value for the dark matter density of the universe. It is around $23 \%$ of the total matter density. Therefore when calculations are plausible, constraints can be put on the different models according to what amount of dark matter they produce. On the other hand, earth-based experiments like CDMS put constraints on dark matter particles because, provided we know the local dark matter density, the nondetection restrains the cross section of those particles scattered off nuclei targets.

The case of dark matter candidates from technicolor theories is not a new subject. Several authors in the past studied the scenario of having a neutral technibaryon as a natural candidate for dark matter [6-8]. Recently it was suggested that technicolor theories that have techniquarks transforming under not the fundamental but under higher representations of the gauge group can be viable extensions of the standard model, because they are within the limits of the electroweak precision measurements and close to the conformal window [9-16]. In the minimal model, only two flavors of techniquarks and an $\mathrm{SU}(2)$ gauge group are sufficient to make the theory quasiconformal. Because the addition of new particles is small, this model is within the electroweak measurements and because of the quasiconformality this model avoids the problems of the old technicolor theories, such as giving mass to the heavy particles like the top quark. The attraction of these models enhances since they can achieve unification of couplings [17]. There can be several different possibilities for having dark matter candidates from these technicolor theories. A first attempt was done in $[15,18,19]$, where the possibility of having a component of dark matter from a neutral pseudo-Goldstone boson technibaryon was investigated. If there are no processes violating the technibaryon number (apart from sphalerons), and there is an initial technibaryon-antitechnibaryon asymmetry and the neutral technibaryon is the lightest one, then it is absolutely stable. This technibaryon with a mass of the order of $\mathrm{TeV}$ can 
account for even the whole dark matter density. However, since in this case the WIMP is a boson, it can scatter coherently off nuclei targets. As a result, the cross section for elastic collision with nuclei targets is 4 times the spin independent one of a heavy Dirac neutrino. Such a large cross section (given we accept that the local dark matter density in the neighborhood of the earth is $0.2-0.4 \mathrm{GeV} / \mathrm{cm}^{3}$ ) should give a considerable number of counts in earth-based experiments like CDMS. The CDMS collaboration has not detected any counts so far [20]. This technibaryon is ruled out as dark matter candidate if it should account for the whole dark matter density. However, if the technibaryon consists of a component of dark matter up to $20 \%$, it cannot yet be ruled out [18].

Another interesting possibility of a dark matter candidate from the same technicolor model was studied in [21]. The dark matter candidate in this scenario is the neutrino of a fourth family of heavy leptons. In the minimal walking technicolor theory with techniquarks transforming under the 2-index symmetric representation of the technicolor gauge group, a fourth family of leptons is needed in order to cancel Witten global anomaly for the SU(2) weak group. If the techniquarks and the fourth family leptons have hypercharge assignments as the corresponding standard model particles, then the fourth neutrino is electrically neutral and it can account for the whole dark matter density if the evolution in the early universe is dominated by the quintessencelike dark energy component constrained by nucleosynthesis.

In this paper we investigate an interesting alternative possibility to the previous scenarios. We study the case of a dark matter candidate made of a compound bound state of a techniquark with a technigluon forming a Majorana particle through a usual seesaw mechanism. Because Majorana fermions cannot interact coherently with the nucleus, such particles have smaller cross section and therefore fewer projected counts in CDMS. We calculate the relic density of these particles and we address the issue of their detection. We should mention that our results are also complementary to the scenario studied in [21] as we shall explain in the next sections.

\section{TECHNICOLOR MODEL AND DARK MATTER CANDIDATE}

The technicolor model we are going to use is the one used in $[15,18,21]$. The technicolor group is an SU(2) and there are just two techniquarks $U$ and $D$ transforming under the adjoint representation of SU(2). The global symmetry of the model is an $S U(4)$ that breaks spontaneously down to an $\mathrm{SO}(4)$ resulting in 9 Goldstone bosons, 3 of which are eaten by the $W$ and $Z$ bosons [15]. The two techniquarks form a doublet under the electroweak gauge symmetry. There are two extra particles, i.e. a "new neutrino" $\nu^{\prime}$ and a "new electron" $\zeta$ coupled to the electroweak in order to cancel the global Witten anomaly. The authors of $[15,18]$ showed that, for a specific assignment of the weak hypercharge for the technicolor particles, which is allowed by the cancellation of gauge anomalies, one of the techniquarks (for example, the $D$ ) is electrically neutral. Therefore the Goldstone technibaryons of the theory made exclusively of $D$ techniquarks, if they are the lightest technibaryons of the theory, can be a legitimate dark matter candidate. As we mentioned in the Introduction, although such a possibility is very natural, the large cross section of the technibaryon scattering off a nuclei target excludes this scenario if the technibaryon consists $100 \%$ of the dark matter density.

In this paper we are going to study a slightly different case. We are going to assume the same hypercharge assignments as in $[15,18]$, so again the $D$ techniquark is electrically neutral, but we are not assuming that the Goldstone technibaryon made of $D$ is the lightest stable object. Rather in this scenario we assume that bound states between $D$ techniquarks and technigluons $G$ are the lightest objects. This is something of course not encountered in QCD, since it is impossible to make a colorless object out of a quark and a gluon. This is because quarks transform under the fundamental representation and gluons under the adjoint representation of the gauge group. However, in this particular technicolor model both techniquarks and technigluons transform under the adjoint representation that makes it possible to form a colorless object. Since we have two colors, red $(r)$ and green $(g)$, in the adjoint representation, we have three color states: $r r, r g+g r$, and $g g$. If we number these states from 1 to 3 , the objects $D_{L}^{\alpha} G^{\alpha}$ and $D_{R}^{\alpha} G^{\alpha}$ are colorless. It is assumed that we have chosen the "appropriate" basis for $G^{\alpha}$ and we sum over $\alpha$ which runs from 1 to 3 . Apparently, similar colorless states can be constructed also using the $U$ techniquark.

Unlike in $[15,18]$, we assume that at the GUT scale, extended technicolor (ETC) interactions violate the technibaryon number. In addition, we do not assume that there is an initial technibaryon asymmetry. It is not necessary to speculate regarding the particular ETC model. It is sufficient for our purpose to assume that below the ETC scale these technibaryon violating processes behave effectively as a Majorana mass term for the left-handed neutral techniquarks. The low energy effective theory has mass terms of the form

$$
\cdots-m_{D}\left(\psi_{L}^{\dagger} \psi_{R}+\psi_{R}^{\dagger} \psi_{L}\right)-\frac{1}{2} M\left(\psi_{L}^{c \dagger} \psi_{L}+\psi_{L}^{\dagger} \psi_{L}^{c}\right),
$$

where $\psi_{L}$ and $\psi_{R}$ are the left- and right-handed Weyl spinors of the technigluon-dressed neutral techniquark. For example, $\psi_{L}$ is the colorless $D_{L}^{\alpha} G^{\alpha}$. The $c$ index denotes charge conjugation, $m_{D}$ is the Dirac mass of the technigluon-dressed techniquarks, and $M$ is the Majorana mass for the left-handed ones. On general grounds we can give a Majorana mass also to the right-handed techniquarks or, for instance, we can give a Majorana mass only to the right-handed and not to the left-handed particles. Although 
not forbidden per se, we shall argue that the case of lefthanded Majorana particles is far more interesting from the point of view of phenomenology. The mass matrix is

$$
L_{\text {mass }}=-\frac{1}{2}\left(\psi_{L}^{\dagger} \psi_{R}^{c \dagger}\right)\left(\begin{array}{cc}
M & m_{D} \\
m_{D} & 0
\end{array}\right)\left(\begin{array}{l}
\psi_{L}^{c} \\
\psi_{R}
\end{array}\right)+\text { H.c. }
$$

The usual seesaw mechanism gives two mass eigenvalues $M_{1}=\left(M+\sqrt{M^{2}+4 m_{D}^{2}}\right) / 2$ and $M_{2}=\left(\sqrt{M^{2}+4 m_{D}^{2}}-\right.$ $M) / 2$ which at the limit where $M \gg m_{D}$ become respectively $M_{1} \simeq M$ and $M_{2} \simeq m_{D}^{2} / M$. The two Majorana particles (that are mass eigenstates) constructed from the leftand right-handed techniquarks are

$$
\begin{gathered}
N_{1}=\cos \theta\left(\begin{array}{l}
\psi_{L} \\
\psi_{L}^{c}
\end{array}\right)+\sin \theta\left(\begin{array}{c}
\psi_{R}^{c} \\
\psi_{R}
\end{array}\right), \\
N_{2}=\sin \theta\left(\begin{array}{c}
i \psi_{L} \\
-i \psi_{L}^{c}
\end{array}\right)+\cos \theta\left(\begin{array}{c}
-i \psi_{R}^{c} \\
i \psi_{R}
\end{array}\right),
\end{gathered}
$$

where the angle $\theta$ is defined through $\tan 2 \theta=2 m_{D} / M$. Varying the angle $\theta$ within $0<\theta<\pi / 4$, we can get the full range of the ratio $m_{D} / M$ from zero $\left(m_{D} \ll M\right)$ to infinity $\left(m_{D} \gg M\right)$. At the limit where $m_{D} \ll M, \tan \theta \simeq$ $m_{D} / M$. Alternatively, we can write the original fields in terms of the particles $N_{1}$ and $N_{2}$,

$$
\begin{aligned}
& \psi_{L}=\cos \theta P_{L} N_{1}-i \sin \theta P_{L} N_{2}, \\
& \psi_{R}=\sin \theta P_{R} N_{1}-i \cos \theta P_{R} N_{2},
\end{aligned}
$$

where $P_{R}$ and $P_{L}$ are the right- and left-handed projection operators $\left(1 \pm \gamma_{5}\right) / 2$. Now let us recall how the gluondressed $D$ techniquark $\psi_{L}$ couples to the weak gauge bosons. Since we have chosen the $D$ techniquark to be electrically neutral, the hypercharge derived from the relation $Q=T_{3}+Y$ must be $1 / 2$. This means that $\psi_{L}$ couples only to the $Z$ boson as

$$
L_{Z}=\frac{\sqrt{g^{2}+g^{12}}}{2} Z_{\mu} \bar{\psi}_{L} \gamma^{\mu} \psi_{L}
$$

For completeness we should mention that the charge conjugated field $\psi_{L}^{c}$ couples to the $Z$ with the same strength but opposite sign. Now we can write how the $Z$ boson couples to the Majorana particles $N_{1}$ and $N_{2}$. Using Eqs. (3)-(5) and (7), we get the following couplings to the $Z$ :

$$
\begin{aligned}
& \frac{\sqrt{g^{2}+g^{12}}}{2} Z_{\mu}\left(\cos ^{2} \theta \bar{N}_{1} \gamma^{5} \gamma^{\mu} N_{1}+\sin ^{2} \theta \bar{N}_{2} \gamma^{5} \gamma^{\mu} N_{2}\right. \\
& \left.\quad+i \sin \theta \cos \theta \bar{N}_{1} \gamma^{5} \gamma^{\mu} N_{2}+\text { H.c. }\right) .
\end{aligned}
$$

It is easy to interpret the above interactions at the limit where $m_{D} \ll M$. Since $N_{1}$ is mostly $\psi_{L}$, it couples strongly to the $Z$, whereas for $N_{2}$ being mostly $\psi_{R}$, the interaction is suppressed by the factor $\sin ^{2} \theta$. It is also evident that the interaction among $N_{1}, N_{2}$ and $Z$ is somewhat suppressed by just one power of $\sin \theta$. Because both $N_{1}$ and $N_{2}$ are
Majorana particles, the technibaryon number is not protected as in the scenario presented in $[15,18]$. This means that two of the $N_{1}$ or $N_{2}$ can annihilate each other. We shall show that the heavy $N_{1}$ decays fast enough so its relic density today is zero. The lighter $N_{2}$ is our dark matter candidate for this scenario. We shall argue that the annihilation cross section for $N_{2}$ is not big enough in order to cause the complete annihilation of its relic density.

As we already mentioned, the U(1) symmetry of the technibaryon number is broken because of the Majorana mass term. However, the lightest technibaryon $\left(N_{2}\right.$ in this scenario) is protected by a $Z_{2}$ symmetry, i.e. the Lagrangian is invariant if $N_{2} \rightarrow-N_{2}$. The $Z_{2}$ symmetry in this case is analogous to the $R$-parity in SUSY protecting the neutralino from decaying. As long as the ETC model respects the $Z_{2}$ symmetry and $N_{2}$ is the lightest technibaryon, $N_{2}$ cannot decay, but coannihilate with another $N_{2}$.

Because of our ignorance regarding the exact ETC model and the nonperturbative nature of the dynamics, it is difficult to conclude decisively that a state of $D G$ can be lighter than the regular technibaryons of the theory. However, studies of Super Yang Mills theories with supersymmetry softly broken showed that a Majorana mass for the gluino $\lambda$ makes the $\lambda G$ lighter than the $\lambda \lambda$ [22]. Although our model is not supersymmetric, this is an encouraging indication that $D G$ might be indeed the lightest technibaryon of the theory.

By inspection of Eqs. (1)-(4), (7), and (8), one can realize that $D_{L} G$ couples to $Z$ with the same strength as a left-handed neutrino. In this analogy $\psi_{L}$ and $\psi_{R}$ correspond to a left- and a right-handed neutrino. Our scenario is analogous to the one studied in [21], where there is one left-handed heavy neutrino that has either Dirac or Majorana mass. Our study is analogous to the case where the heavy left-handed neutrino has both Majorana and Dirac mass. From this point of view, $N_{1}$ and $N_{2}$ are two Majorana neutrinos. Therefore our results for the relic density and the CDMS and LEP constraints are directly applicable in this case also.

\section{RELIC DENSITY OF THE TECHNICOLOR WIMP}

During the past few years we have obtained a lot of information regarding the baryon and dark matter density from WMAP. The current knowledge is that $\Omega \simeq 1$ with the baryon density being $\Omega_{B} h^{2}=0.022$ and dark matter density $\Omega_{d} h^{2}=0.112$ [23]. In this section of the paper we calculate the relic density of the Majorana particle $N_{2}$ and we show that it can account for the full dark matter density for a range of masses and of the angle $\theta$. The relic density of such a particle is governed by the well-known Boltzmann equation,

$$
\frac{d n_{N_{2}}}{d t}+3 H n_{N_{2}}=-\left\langle\sigma_{A} v\right\rangle\left[\left(n_{N_{2}}\right)^{2}-\left(n_{N_{2}}^{\mathrm{eq}}\right)^{2}\right],
$$


where $n_{N_{2}}$ and $n_{N_{2}}^{\mathrm{eq}}$ are the number density of $N_{2}$ at time $t$ and at equilibrium, respectively, $H$ is the Hubble expansion rate, and $\left\langle\sigma_{A} v\right\rangle$ is the thermally averaged cross section for $N_{2} N_{2}$ annihilation times the relative velocity. On general grounds the annihilation cross section should have the velocity dependence $v^{p}$. The value $p=0$ corresponds to $s$-wave annihilation and $p=2$ corresponds to a $p$-wave annihilation. Indeed this is the case for Majorana particles. The thermal velocity is $\left\langle v^{2}\right\rangle \sim T / m$. Therefore we can write the annihilation cross section times the relative velocity as

$$
\left\langle\sigma_{A} v\right\rangle=\sigma_{0}(T / m)^{n}=\sigma_{0} x^{-n},
$$

where $m$ is the mass of $N_{2}, T$ is the temperature, and $x=$ $m / T$ [24]. It is understood that the $s$-wave annihilation corresponds to $n=0$ and the $p$-wave one corresponds to $n=1$. The Boltzmann equation can be rewritten in a more convenient form in terms of $Y=n_{N_{2}} / s$, ( $s$ being the entropy density) as

$$
\frac{d Y}{d x}=-\lambda x^{-n-2}\left(Y^{2}-Y_{\mathrm{eq}}^{2}\right)
$$

where

$$
\lambda=0.264\left(g_{* s} / g_{*}^{1 / 2}\right) M_{P l} m \sigma_{0} .
$$

We define $Y_{\text {eq }}=n_{N_{2}}^{\text {eq }} / s, M_{P l}=1.22 \times 10^{19} \mathrm{GeV}$. The $g_{*}$ and $g_{* s}$ are dimensionless numbers defined in [24]. Roughly speaking, they count the total number of effectively massless degrees of freedom. For energies above $1 \mathrm{MeV}, g_{*}$ and $g_{* s}$ are practically identical. At a temperature of $1 \mathrm{GeV}, g_{*}$ and $g_{* s}$ are about 80 , increasing mildly to roughly 100 as temperature increases up to $1 \mathrm{TeV}$. The knowledge of the annihilation cross section and the mass of $N_{2}$ is sufficient enough to determine the relic density of $N_{2}$.

The $N_{2}$ couples to the $Z$ as it can be seen from Eq. (8) as a Majorana neutrino times $\sin ^{2} \theta$. There are two general cases regarding the annihilation cross section of $N_{2}$. The first case is when the mass of $N_{2}$ is smaller than the mass of the $W$ boson and the other one when the mass is larger. We investigate separately the two cases because different annihilation channels contribute to each of them.

\section{A. $\boldsymbol{m}<M_{W}$}

In this case the annihilation of two $N_{2}$ occurs into pairs of light fermion-antifermion (as, for example, light neutrino-antineutrino pair or electron-positron pair) through $Z$ exchange. We calculated the average cross section times the relative velocity for annihilation of two $\mathrm{N}_{2}$ into a pair of fermion-antifermion which is in accordance with [24]

$$
\left\langle\sigma_{A} v\right\rangle=\frac{4 G_{F}^{2} m^{2}}{3 \pi}\left\langle\beta^{2}\right\rangle\left(C_{V}^{2}+C_{A}^{2}\right) \sin ^{4} \theta,
$$

where $G_{F}$ is the Fermi constant, and $\beta$ is the velocity of $N_{2}$ at the center of mass reference system. The parameters $C_{V}$ and $C_{A}$ are defined as $C_{V}=j_{3}-2 q \sin ^{2} \theta_{w}$ and $C_{A}=j_{3}$, where $j_{3}$ and $q$ are, respectively, the weak isospin and the electric charge of the fermion and $\sin \theta_{w}$ is the Weinberg angle. For the total annihilation cross section, we should include all possible channels with fermions that are lighter than $N_{2}$. For a mass of $N_{2}$ larger than $5 \mathrm{GeV}$, the number of open channels for annihilation into pairs of fermionantifermion includes all leptons and all quarks (times three colors) except the top one $[25,26]$. The total annihilation cross section can be written as

$$
\left\langle\sigma_{A} v\right\rangle=N \frac{2 G_{F}^{2} m^{2}}{3 \pi}\left\langle\beta^{2}\right\rangle \sin ^{4} \theta,
$$

where $N=14.47$ represents the effective number of channels. In principle, $N$ should have been 21 since we include five quarks times three colors and six leptons. However, since all the fermions do not couple with the same strength to the $Z$, the total annihilation cross section is equivalent to the total cross section of $N$ channels of neutrinoantineutrino. For the derivation of the cross section, we assumed that the fermions are much lighter than $N_{2}$. Equation (14) is valid only in the case where $m \ll M_{Z}$. For larger values of $m$ we must take into account the resonance effect and the fact that the denominator of the propagator of the virtual $Z$ boson is not anymore dominated by the mass of the $Z$. In this case (14) must be modified as

$$
\left\langle\sigma_{A} v\right\rangle=N \frac{2 G_{F}^{2} m^{2}}{3 \pi}\left\langle\beta^{2}\right\rangle \sin ^{4} \theta \frac{M_{Z}^{4}}{\left(s-M_{Z}^{2}\right)^{2}+\Gamma_{Z}^{2} M_{Z}^{2}},
$$

where $\Gamma_{Z}=2.5 \mathrm{GeV}$ is the width of the $Z$ and $s$ is the Mandelstam variable which at the nonrelativistic limit is $s \simeq 4 \mathrm{~m}^{2}$. In principle, one can argue that particles like $N_{2}$ that couple to the $Z$ boson with a mass of a few $\mathrm{GeV}$ are already excluded by constraints from the measurement of the width of the $Z$ by the LEP collaboration. In fact, a fourth neutrino coupled to the $Z$ with the same strength as the other three ones has been excluded by the LEP collaboration for a mass up to $40-45 \mathrm{GeV}$ [27]. However in this case, $N_{2}$ can avoid exclusion by LEP if the angle $\theta$ is small. We can see from Eq. (8) that $N_{2}$ couples to the $Z$ as a regular neutrino times $\sin ^{2} \theta$. Therefore if $\theta$ is sufficiently small then $N_{2}$ cannot be excluded by LEP even for masses smaller than $40 \mathrm{GeV}$. We address this question later on this subsection. A similar case regarding neutrinos was studied in [28]. Another constraint is provided by earth-based experiments for dark matter search like CDMS. However, as we shall show in the next section, the elastic cross section of $N_{2}$ scattering off the nuclei of the detectors is very small to be ruled out by CDMS.

In order to calculate the relic density, we have to solve Eq. (11). A very good approximate solution for nonrelativistic particles has been given pedagogically in $[24,29]$. The approximate solution for $Y$ is 


$$
Y_{\infty}=\frac{3.79(n+1) x_{f}^{n+1}}{\left(g_{* s} / g_{*}^{1 / 2}\right) M_{P l} m \sigma_{0}},
$$

where $x_{f}$ denotes the value of $x$ where the decoupling occurs. The value of $x_{f}$ is given by the approximate relation

$$
x_{f} \simeq \ln [(2+c) c \lambda \alpha]-\left(n+\frac{1}{2}\right) \ln [\ln [(2+c) c \lambda \alpha]] .
$$

The parameter $c$ is a fitting numerical constant of order unity. Usually the best fitting to the real solution is achieved when $c(c+2)=n+1$. The parameter $\alpha=$ $0.145\left(\mathrm{~g} / \mathrm{g}_{* s}\right)$, where $g$ is the number of degrees of freedom for the particle $N_{2}$ (therefore $g=2$ ). The relic abundance is

$$
\Omega_{N_{2}} h^{2}=Y_{\infty} s m /\left(\rho_{\text {crit }} / h^{2}\right) \simeq 2.82 \times 10^{8} Y_{\infty}(m / \mathrm{GeV}) .
$$

By inspection of (15) we conclude that $n=1$ and

$$
\sigma_{0}=\frac{N G_{F}^{2} m^{2} \sin ^{4} \theta}{\pi} \frac{M_{Z}^{4}}{\left(s-M_{Z}^{2}\right)^{2}+\Gamma_{Z}^{2} M_{Z}^{2}} .
$$

This is because the thermal average velocity at the center of mass reference system is given by

$$
\left\langle\beta^{2}\right\rangle=\frac{3}{2} \frac{T}{m} .
$$

It is easy to prove the above relation if one notices that the thermal average velocity in the lab frame is related to the one at the center of mass frame as $\left\langle\beta_{\text {lab }}^{2}\right\rangle=2\left\langle\beta^{2}\right\rangle$. By using the equipartition theorem, we get $\left\langle\beta_{\text {lab }}^{2}\right\rangle=3(T / m)$ and therefore $\left\langle\beta^{2}\right\rangle$ is given by (20). Using Eqs. (18) and (19) and a value $g_{*}=100$, we calculated the relic density of $N_{2}$. For $m \ll M_{Z}$ the expression takes the simple form

$$
\Omega_{N_{2}} h^{2}=\frac{0.0283 x_{f}^{2}}{m^{2} \sin ^{4} \theta} .
$$

This relation is slightly more complicated once we include the extra term of (15) compared to (14). In Fig. 1 we show the value of $\sin \theta$ that gives the proper relic density for $N_{2}$ (if it accounts for the whole dark matter) as a function of its mass, for a range of $m$ from 10 to $80 \mathrm{GeV}$. For a mass of $10 \mathrm{GeV}$, the dark matter density is achieved for $\sin \theta=1$. For a mass lower than $10 \mathrm{GeV}, N_{2}$ has a relic density larger than the dark matter density $\Omega_{d} h^{2}=0.112$. If we increase the mass, $\sin \theta$ drops, reaching 0.08 for $m=45.5 \mathrm{GeV}$, which is half of $M_{Z}$. As $m$ increases beyond the resonant value, the annihilation cross section decreases and a higher value of $\sin \theta$ is needed in order to maintain $\Omega_{N_{2}} h^{2}=$ 0.112 . We have plotted $\sin \theta$ up to $80 \mathrm{GeV}$, which is the onset of a new dominant channel that we examine in the next subsection. Since $0<\theta<\pi / 4, \sin \theta$ is restricted between $0<\sin \theta<\sqrt{2} / 2=0.707$. It is evident from Fig. 1 that for $m<18 \mathrm{GeV}$ where $\sin \theta>0.707, N_{2}$ cannot provide the dark matter density and this region is excluded.

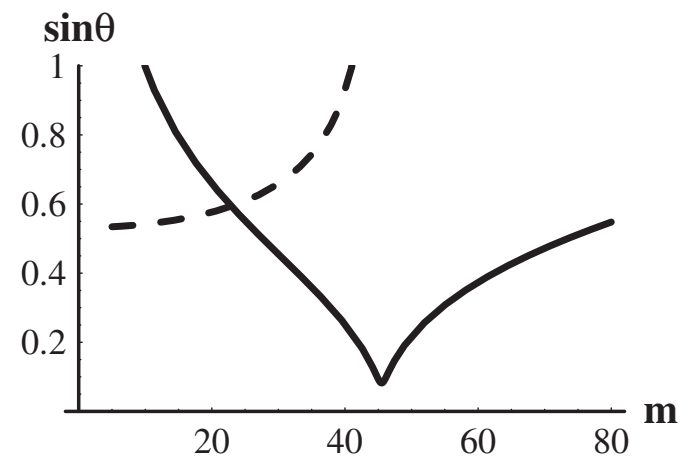

FIG. 1. The solid line shows the dependence of $\sin \theta$ on the mass of $N_{2}$ (in $\mathrm{GeV}$ ), in order the relic density $\Omega_{N_{2}} h^{2}=0.112$. The dashed line shows the constraint on $m$ and $\sin \theta$ imposed by LEP. The area above the dashed line is excluded. This means that $m$ should be larger than $23 \mathrm{GeV}$, which is the value where the two curves cross each other.

This region is also excluded by LEP as we show in the next paragraph.

There are constraints on the masses of neutral particles that couple to the $Z$ boson imposed by the LEP experiment. In LEP the total decay width for the $Z$ boson into invisible neutral particles was measured with very high accuracy. The ratio of the decay width into invisible particles over the decay rate into a pair of neutrino-antineutrino determines the number of light neutral particles coupled to the $Z$. The experimental value of this ratio is [27]

$$
N_{\nu}=\frac{\Gamma(Z \rightarrow \text { invisible })}{\Gamma(Z \rightarrow \bar{\nu} \nu)}=3.00 \pm 0.08
$$

We interpret the bound as implying that the number of light species is $N_{\nu}<3.08$. The constraint for $N_{2}$ can be written as

$$
0.08>N_{\nu}-3=\sin ^{4} \theta \times \beta^{3},
$$

where $\beta$ is the velocity of $N_{2}$ produced as $Z$ decays [30]. In Fig. 1 we implemented this constraint. As it was expected, low masses up to $23 \mathrm{GeV}$ are excluded by LEP. However, we can see in the figure that LEP cannot exclude the region above $23 \mathrm{GeV}$. For a typical value $m=40 \mathrm{GeV}$, the mass of $N_{1}$ is $m_{N_{1}} \simeq 589 \mathrm{GeV}$. This corresponds to a Majorana mass $M \simeq 549 \mathrm{GeV}$ and a Dirac mass $m_{D} \simeq 153 \mathrm{GeV}$. In the next section we shall address the issue of $N_{2}$ detection by the CDMS experiment. We shall argue that CDMS imposes no further constraints on the suppression angle $\sin \theta$. For completeness we also checked if it is possible for the heavier $N_{1}$ particle to sustain any considerable relic density. From (8) we can calculate the decay rate of $N_{1}$ to an $N_{2}$ and a $Z$. In order for a particle to give a considerable relic density, the decay rate has to be smaller than the Hubble parameter. The decay rate of $N_{1}$ is proportional to 


$$
\left(\frac{\sqrt{g^{2}+g^{\prime 2}}}{2}\right)^{2} \sin ^{2} \theta \cos ^{2} \theta \frac{M_{N_{1}}^{3}}{M_{Z}^{2}} .
$$

The formula is similar to the decay rate of the top quark. The Hubble parameter has an extremely low value of $\sim 10^{-33} \mathrm{eV}$. For any realistic value of $M_{N_{1}}$, and unless there is no extreme fine-tuning of the mass difference among $N_{1}, N_{2}$, and $Z$ or of the $\sin ^{2} \theta \cos ^{2} \theta$ factor, it is impossible for the decay rate of $N_{1}$ to be smaller than the Hubble parameter. Therefore there is no relic density for $N_{1}$ since it decays very fast to $N_{2}$ and $Z$.

\section{B. $\boldsymbol{m}>M_{W}$}

The second case we investigate is the one where $m>$ $M_{W}$. In principle, this means that we examine the possibility of $m$ being higher than $80 \mathrm{GeV}$. No constraints are imposed by the LEP experiment on this regime since the mass is higher than half of $M_{Z}$. In order to calculate the relic abundance, we use again the Boltzmann Eq. (11). However, the annihilation cross section is different in this case. It is very easy to show that the annihilation of two Majorana $\mathrm{N}_{2}$ into pairs of light fermions (like electronpositron or quark-antiquark) for $m>M_{Z}$ is suppressed by a factor $(1 / 16)\left(M_{Z} / m\right)^{4}$. This is because the propagator of the virtual $Z$ boson is $1 /\left(q^{2}-M_{Z}^{2}\right)$. In the case of $m \ll M_{Z}$ the propagator scales approximately as $1 / M_{Z}^{2}$. However, if $m \gg M_{Z}$ the propagator scales as $1 / s \simeq 1 /\left(4 m^{2}\right)$. The cross section depends on the square of the propagator and therefore the cross section is suppressed by the factor we mentioned above. In this regime a new channel opens up and becomes the dominant one [31]. It is the annihilation into a pair of $W^{+}-W^{-}$through a $Z$ boson. We calculated the cross section and we found

$$
\begin{aligned}
\left\langle\sigma_{A} v\right\rangle= & \frac{G_{F}^{2} m^{2}}{3 \pi} \beta^{2} \beta_{W} \frac{s^{2}}{\left(s-M_{Z}^{2}\right)^{2}+\Gamma_{Z}^{2} M_{Z}^{2}} \\
& \times \sin ^{4} \theta\left(1-\mathcal{O}\left(\frac{M_{W}^{2}}{m^{2}}\right)\right) .
\end{aligned}
$$

Again $\beta$ is the velocity of $N_{2}$ at the center of mass frame and $\beta_{W}=\sqrt{1-4 M_{W}^{2} / s}$ is the velocity of the $W$. Using (10) as in the previous case we can write $\sigma_{0}$ as

$$
\sigma_{0}=\frac{G_{F}^{2} m^{2}}{2 \pi} \beta_{W} \frac{s^{2}}{\left(s-M_{Z}^{2}\right)^{2}+\Gamma_{Z}^{2} M_{Z}^{2}} \sin ^{4} \theta\left(1-\mathcal{O}\left(\frac{M_{W}^{2}}{m^{2}}\right)\right) .
$$

At the limit where $m \gg M_{W}$, the above equation takes the simple form

$$
\sigma_{0}=\frac{G_{F}^{2} m^{2}}{2 \pi} \sin ^{4} \theta
$$

At the same limit the relic abundance of $N_{2}$ can be written as

$$
\Omega_{N_{2}} h^{2}=\frac{0.818 x_{f}^{2}}{m^{2} \sin ^{4} \theta} .
$$

In Fig. 2 we plot the dependence of $\sin \theta$ as a function of the mass $m$ from $80 \mathrm{GeV}$ up to $2 \mathrm{TeV}$ in order to get a relic density $\Omega_{N_{2}} h^{2}=0.112$. In our plot we took into account both the annihilation channel to $W^{+}-W^{-}$and to pairs of fermions-antifermions. For the $W^{+}-W^{-}$channel we dropped the terms that scale as powers of $\left(M_{W} / m\right)^{2}$. The mixing angle $\sin \theta$ has a peak at $122 \mathrm{GeV}$ and then it drops smoothly as $m$ increases. It is easy to see why this peak appears. As soon as $m$ becomes larger than $80 \mathrm{GeV}$, it is possible to have annihilation to a pair of $W^{+}-W^{-}$. However close to the onset, the phase space for this amplitude is very small and the cross section is controlled by $\beta_{W}$ (which is zero at $s=4 M_{W}^{2}$ ). Between 80 and $122 \mathrm{GeV}$, the total annihilation cross section drops because the $W^{+}-W^{-}$channel has not yet enough phase space and the fermionantifermion channels that still dominate have a cross section that falls as we explained at the beginning of this subsection. Once $m$ becomes large enough so there is a lot of phase space for the $W^{+}-W^{-}$annihilation, the cross section increases. This means that $\sin \theta$ must drop if we have to maintain the dark matter density. At a mass of $1 \mathrm{TeV} \sin \theta=0.26$. For this mass of $N_{2}$, the corresponding mass for the heavy $N_{1}$ is $13.5 \mathrm{TeV}$ and the original Dirac and Majorana masses are respectively $m_{D}=3.7 \mathrm{TeV}$ and $M=12.5 \mathrm{TeV}$. We plot $\sin \theta$ up to $m=2 \mathrm{TeV}$ where $\sin \theta=0.19$. Our calculation of the total cross section and consequently of the value of $\sin \theta$ is extremely accurate both at the onset of the $W^{+}-W^{-}$channel and for $m \gg M_{W}$. The only region where the cross section is not very accurate is at the peak (around $122 \mathrm{GeV}$ ) because this is where the corrections of the order of $M_{W}^{2} / \mathrm{m}^{2}$ are important. For masses larger than $122 \mathrm{GeV}$, these corrections are suppressed. For masses close to the onset of the $W^{+}-W^{-}$ channel, these corrections are unimportant because the annihilation cross section is still dominated by the annihilation to pairs of fermions-antifermions. However, even at

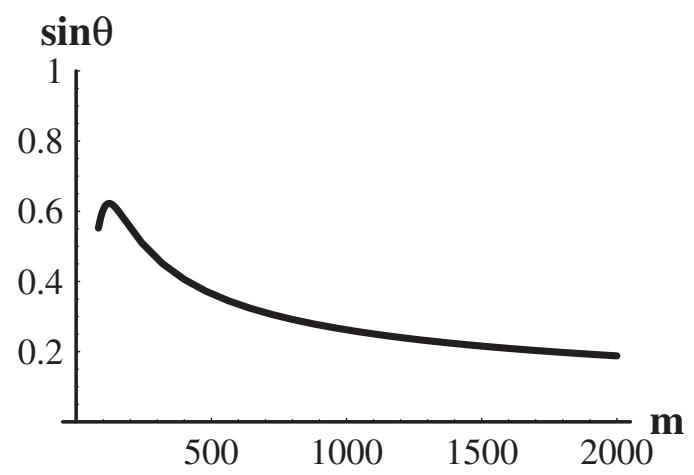

FIG. 2. As in Fig. 1, the solid line shows the dependence of $\sin \theta$ on the mass of $N_{2}$ (in $\mathrm{GeV}$ ), in order the relic density $\Omega_{N_{2}} h^{2}=0.112$. 
the peak, our estimation for the $\sin \theta$ is off at most by $\sim 10 \%$. Either on the left or on the right of the peak our estimation of $\sin \theta$ becomes better than $95 \%$ accurate within a few $\mathrm{GeV}$.

\section{DETECTION OF THE LIGHTEST TECHNIBARYON IN CDMS}

We turn our attention now to the question of detection of $\mathrm{N}_{2}$ from dark matter search experiments. It is well known that earth-based experiments like CDMS can put tight constraints regarding the cross section of WIMPs scattering off nuclei targets. In fact, the most important constraint related to the scenario of the techni-Goldstone boson dark matter candidate was coming from the nondetection of counts in CDMS $[15,18]$. There are two basic factors that influence the number of counts on earth detectors. The first one is the local dark matter density and the second one is the elastic scattering cross section between the WIMP and the nuclei of the detector. Most cosmologists agree that the local dark matter density should be somewhere between $0.2-0.4 \mathrm{GeV} / \mathrm{cm}^{3}$. As for the cross section, Majorana particles have usually much smaller cross section compared to Dirac ones because Majorana fermions do not scatter coherently with the whole nucleus of the target. This is our motivation for investigating $N_{2}$ as a dark matter candidate. A review of the cross section of different dark matter candidates can be found in [32,33]. For a Majorana particle only spin-dependent elastic collisions contribute $[34,35]$. Following [34], we can write the spin-dependent cross section for $N_{2}$ as

$$
\sigma_{N_{2}}=\frac{2 G_{F}^{2}}{\pi} \mu^{2} I_{S} \sin ^{4} \theta,
$$

where $\mu$ is the reduced mass of the system WIMP-nucleus and $I_{s}$ is conventionally written in the form $I_{s}=$ $C^{2} \lambda^{2} J(J+1) . C$ is given by

$$
C=\sum_{q} T_{q}^{3} \Delta_{q} \quad(q=u, d, s),
$$

where $\Delta_{q}$ is the fraction of the spin carried by the specific quark $q . T_{q}^{3}$ is the 3 rd component of the isotopic spin of each of the three quarks $\left(T_{u}^{3}=1 / 2, T_{d}^{3}=-1 / 2, T_{s}^{3}=\right.$ $-1 / 2)$. The values for the different $\Delta_{q}$ given by the European Muon Collaboration are $\Delta_{u}=0.83, \Delta_{d}=$ -0.43 , and $\Delta_{s}=-0.10$ [34]. A realistic value for $\lambda^{2} J(J+1)$ within the model of odd group for the detectors of $\mathrm{Ge}^{73}$ is 0.065 . Given these values, the overall factor $I_{s} \simeq$ 0.03 for the Ge detectors. The cross section can be written in convenient units $\mathrm{pb}$ as

$$
\begin{aligned}
\sigma_{N_{2}} & =3.38 \times 10^{-2} \mu^{2} I_{s} \sin ^{4} \theta \\
& =1.01 \times 10^{-3} \mu^{2} \sin ^{4} \theta(\mathrm{pb}) .
\end{aligned}
$$

The total rate of counts on an earth-based detector in experiments like CDMS is [34]

$$
\begin{aligned}
R_{0}= & \frac{540}{A m}\left(\frac{\sigma_{0}}{1 \mathrm{pb}}\right)\left(\frac{\rho_{d m}}{0.4 \mathrm{GeV} \mathrm{c}^{-2} \mathrm{~cm}^{-3}}\right) \\
& \times\left(\frac{v_{0}}{230 \mathrm{kms}^{-1}}\right) \mathrm{kg}^{-1} \text { days }^{-1},
\end{aligned}
$$

where $A$ is the mass number of the nucleus of the detector, $\rho_{d m}$ is the local dark matter density, and $v_{0}$ is the average velocity of the WIMP. The total rate is given in terms of $\mathrm{kg}^{-1}$ days ${ }^{-1}$ which means that, for a given detector of mass $x$ and of exposure time $y$, the total rate must be multiplied by $x y$. However, the number of actual counts that can be seen in a detector is given by

$$
\text { counts }=\frac{d R}{d T} \Delta T \times \tau,
$$

where $\tau$ is the exposure of the detector measured in $\mathrm{kg}$. days and $\Delta T$ is the energy resolution of the detector. The factor $d R / d T$ is the derivative of the total rate with respect to the recoil energy $T$ given by the approximate relation

$$
\frac{d R}{d T}=c_{1} \frac{R_{0}}{E_{0} r} e^{-c_{2} T / E_{0} r},
$$

where $E_{0}$ is the kinetic energy of the WIMP and $r=$ $4 m M_{n} /\left(m+M_{n}\right)^{2}, m$ and $M_{n}$ being the masses of the WIMP and the nucleus of the detector, respectively. The $c_{1}$ and $c_{2}$ are fitting parameters. Equation (34) was derived in [34] after averaging over the Boltzmann velocity distribution of the WIMP. The case with $c_{1}=c_{2}=1$ corresponds to averaging of the velocity from zero to infinity. However, it has been pointed out that the motion of the earth should be taken into account and more realistic values for the parameters are $c_{1}=0.751$ and $c_{2}=0.561$. These parameters depend mildly on the detector's energy threshold and the mass of the WIMP, however do not change a lot and we consider them as constants. We have taken the velocity of the earth to be $v_{E}=1.05 \times v_{0}=$ $1.05 \times 230 \mathrm{~km} / \mathrm{sec}$. In the first results of the CDMS experiment [20], the exposure of the Ge detectors was $19.4 \mathrm{~kg} \cdot$ days. The energy resolution $\Delta T=1.5 \mathrm{keV}$ and the recoil energy threshold is $20 \mathrm{keV}$ although the detector can count recoil energies down to $10 \mathrm{keV}$. The current exposure of the detectors in CDMS $(19.4 \mathrm{~kg} \cdot$ days $)$ is not sufficient to give any counts for a particle like $N_{2}$ with local dark matter density ranging between $0.2-0.4 \mathrm{GeV} / \mathrm{cm}^{3}$. This is true for the whole range of $m$ we examined. In Fig. 3 we show what is the required exposure in order to detect one count of $\mathrm{N}_{2}$ with $90 \%$ confidence level as a function of $m$. The $90 \%$ confidence level corresponds to 2.3 counts. For the first case we studied with $m$ up to $80 \mathrm{GeV}$, the required exposure increases as a function of $m$ up to the resonance peak of $m=$ $45.5 \mathrm{GeV}$ and then drops. For a local dark matter density $\rho=0.4 \mathrm{GeV} / \mathrm{cm}^{3}$, a total amount of $7004 \mathrm{~kg} \cdot$ days is needed for a typical mass $m=30 \mathrm{GeV}$. This practically means that the required exposure for detection of a single 

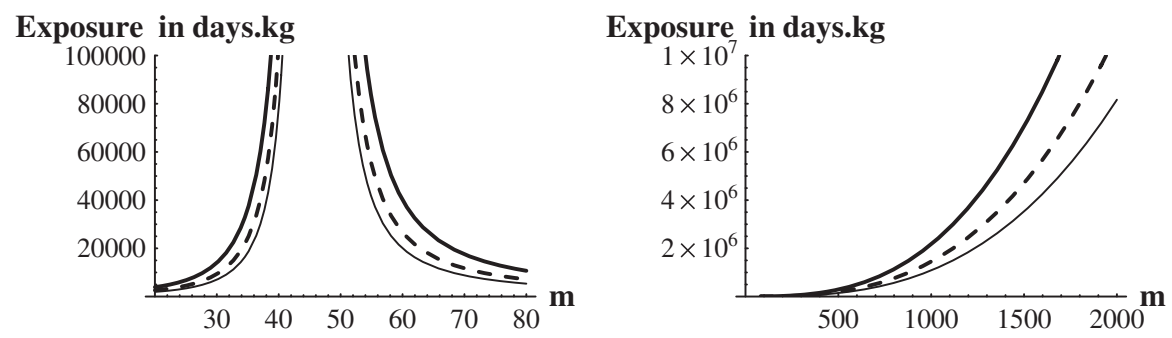

FIG. 3. Left panel: The required exposure of the Ge detectors in $\mathrm{kg} \cdot$ days for a single count (with $90 \%$ confidence level) as a function of $m$ (in $\mathrm{GeV}$ ) for the range $20<m<80$, although in reality $m$ is constrained by LEP to be larger than $23 \mathrm{GeV}$. The thin solid line corresponds to local dark matter density $\rho=0.4 \mathrm{GeV} / \mathrm{cm}^{3}$, the dashed one to $\rho=0.3 \mathrm{GeV} / \mathrm{cm}^{3}$, and the thick solid one to $\rho=$ $0.2 \mathrm{GeV} / \mathrm{cm}^{3}$. For the purposes of presentation, we show the required exposure up to $100000 \mathrm{~kg} \cdot$ days. Around the resonance, where $m=45.5 \mathrm{GeV}$, the required exposure has a sharp peak of about $10^{7} \mathrm{~kg} \cdot$ days. Right panel: As in the left panel for $80<m<$ $2000 \mathrm{GeV}$.

count should be 361 times the current exposure of $19.4 \mathrm{~kg}$ • days. For masses $m \gg 100 \mathrm{GeV}$, the required factor is much larger. For $m=2 \mathrm{TeV}$, the required exposure reaches $8 \times 10^{6} \mathrm{~kg} \cdot$ days. Our results are in accordance with the predictions of the CDMS group for Majorana dark particles [36].

\section{THE CASE OF MAJORANA MASS FOR THE RIGHT-HANDED PARTICLE}

So far we discussed the case of a Majorana mass for the left-handed $D_{L} G$ and a Dirac mass for both $D_{L} G$ and $D_{R} G$. However, one might ask the question of what happens if, instead of giving a Majorana mass to the left-handed particle, we give it to the right-handed one $\left(D_{R} G\right)$. This means that in the mass matrix of Eq. (2), $M$ and 0 in the diagonal are exchanged. It turns out that the two Majorana eigenstates can be described easily using Eqs. (3) and (4) if we make the substitution $\sin \theta \rightarrow \cos \theta$ and $\cos \theta \rightarrow \sin \theta$. Under this description, the relation that defines the angle $\theta$, $\tan 2 \theta=2 m_{D} / M$ remains the same. In addition, the ratio of the masses of $N_{1}$ and $N_{2}$ is given by

$$
\frac{M_{N_{2}}}{M_{N_{1}}}=\tan ^{2} \theta,
$$

as in the previous case. Using this description, $N_{1}$ couples to the $Z$ with a factor $\sin ^{2} \theta$ and $N_{2}$ with a factor $\cos ^{2} \theta$. Since $N_{2}$ is lighter than $N_{1}$, we can find the value of $\cos \theta$ as a function of $M_{N_{2}}$ in order to have $\Omega_{N_{2}} h^{2}=0.112$. This is exactly what we did in the previous sections apart from the fact that the annihilation cross section for $N_{2}$ is not proportional now to $\sin ^{4} \theta$, but $\cos ^{4} \theta$. This means that Figs. 1 and 2 are valid in this case if we substitute in the vertical axis of the figures $\sin \theta$ by $\cos \theta$. However, because (35) remains unchanged, in order for $N_{2}$ to be lighter than $N_{1}$, $\sin \theta<\cos \theta$. This happens when $\cos \theta>\sqrt{2} / 2 \simeq 0.707$. By inspection of Figs. 1 and 2, one can see that there is only one region where this is true. It is below $18 \mathrm{GeV}$ (as seen in Fig. 1) and it is already excluded by LEP.
The physical reason of the qualitative difference between the two general cases we studied, namely, giving a Majorana mass to either the left- or the right-handed particles, relies on the simple fact that in the first case the lighter Majorana is also the particle with the suppressed annihilation cross section and therefore the one that can provide a considerable abundance. In the second case, the lighter Majorana is the one that is "mostly" left-handed and therefore the big annihilation cross section cannot make this particle to sustain a substantial relic density.

\section{CONCLUSIONS AND DISCUSSION}

In this paper we investigated the possibility of a dark matter candidate emerging from the minimal walking technicolor theory. Because the two techniquarks of the theory transform under the adjoint representation of the technicolor $\mathrm{SU}(2)$ group, it is possible to have a bound colorless state between a techniquark and a technigluon. We looked upon the scenario that the left-handed technigluon-dressed techniquark has a Majorana mass and both left- and right-handed have a Dirac mass. We found that this dark matter candidate can account for the whole dark matter density for practically any mass higher than $23 \mathrm{GeV}$. This dark matter candidate can account for the whole dark matter density without being ruled out by LEP or CDMS. We also commented on what happens if it is the right-handed particles that have a Majorana mass instead of the left-handed. We showed that in this case the lighter Majorana particle cannot account for the whole dark matter density. Since we are lacking the tools to calculate the spectrum of this technicolor theory and we do not know the exact ETC model, we cannot know $a$ priori what is the mass of $N_{2}$. Lattice methods just started being implemented for studying the dynamics of models with fermions in higher representations of the gauge group than just fundamental. It will be very interesting if it will be possible to study in lattice this bound state of quark-gluon.

We should emphasize here that our results are complementary to the case studied in [21]. We already mentioned 
that, in the minimal walking technicolor model with techniquarks in the adjoint representation, it is necessary to have an extra family of leptons to cancel Witten global anomaly. If the hypercharge assignment for the fourth neutrino is like in the standard model, then this heavy fourth neutrino can play the role of a dark particle. However, in the candidate we studied, we use a different hypercharge assignment, the one that makes $D$ neutral. Both assignments are consistent and free of gauge anomalies. Although the hypercharge assignments are different, the strength of how $D_{L} G$ couples to the $Z$ boson is the same as this of the fourth neutral neutrino. Therefore if one assumes that the fourth left-handed neutrino (coming from technicolor) has both Majorana and Dirac mass, then the calculation of the relic density and the constraints from LEP and CDMS are identical with the corresponding ones of $\mathrm{N}_{2}$.

\section{ACKNOWLEDGMENTS}

I would like to thank F. Sannino for carefully reading the manuscript and for discussions. I am also grateful to $\mathrm{K}$. Tuominen, and Professor J. D. Vergados and Professor M. Khlopov for their comments. The work of C.K. is supported by the Marie Curie Excellence Grant under Contract No. MEXT-CT-2004-013510.
[1] C. Alcock et al. (MACHO Collaboration), Astrophys. J. 542, 281 (2000).

[2] S. L. Glashow, arXiv:hep-ph/0504287.

[3] D. Fargion, M. Khlopov, and C. A. Stephan, Classical Quantum Gravity 23, 7305 (2006).

[4] D. Fargion and M. Khlopov, arXiv:hep-ph/0507087.

[5] M. Y. Khlopov, Pisma Zh. Eksp. Teor. Fiz. 83, 3 (2006) [JETP Lett. 83, 1 (2006)].

[6] S. Nussinov, Phys. Lett. 165B, 55 (1985).

[7] S. Nussinov, Phys. Lett. B 279, 111 (1992).

[8] S. M. Barr, R. S. Chivukula, and E. Farhi, Phys. Lett. B 241, 387 (1990).

[9] F. Sannino and K. Tuominen, Phys. Rev. D 71, 051901 (2005).

[10] D. K. Hong, S. D. H. Hsu, and F. Sannino, Phys. Lett. B 597, 89 (2004).

[11] D. D. Dietrich, F. Sannino, and K. Tuominen, Phys. Rev. D 72, 055001 (2005).

[12] D. D. Dietrich, F. Sannino, and K. Tuominen, Phys. Rev. D 73, 037701 (2006).

[13] F. Sannino, Int. J. Mod. Phys. A 20, 6133 (2005).

[14] N. Evans and F. Sannino, arXiv:hep-ph/0512080.

[15] S. B. Gudnason, C. Kouvaris, and F. Sannino, Phys. Rev. D 73, 115003 (2006).

[16] D. D. Dietrich and F. Sannino, Phys. Rev. D 75, 085018 (2007).

[17] S. B. Gudnason, T. A. Ryttov, and F. Sannino, arXiv:hepph/0612230 [Phys. Rev. D (to be published)].

[18] S. B. Gudnason, C. Kouvaris, and F. Sannino, Phys. Rev. D 74, 095008 (2006).

[19] E. Accomando et al., arXiv:hep-ph/0608079.
[20] D. S. Akerib et al. (CDMS Collaboration), Phys. Rev. Lett. 93, 211301 (2004).

[21] K. Kainulainen, K. Tuominen, and J. Virkajarvi, Phys. Rev. D 75, 085003 (2007).

[22] N. J. Evans, S. D. H. Hsu, and M. Schwetz, arXiv:hep-th/ 9707260.

[23] D. N. Spergel et al., arXiv:astro-ph/0603449.

[24] E. W. Kolb and M. S. Turner, Front. Phys. 69, 1 (1990).

[25] B. W. Lee and S. Weinberg, Phys. Rev. Lett. 39, 165 (1977).

[26] K. A. Olive and M. S. Turner, Phys. Rev. D 25, 213 (1982).

[27] S. Eidelman et al. (Particle Data Group), Phys. Lett. B 592, 1 (2004).

[28] K. Enqvist and K. Kainulainen, Phys. Lett. B 264, 367 (1991).

[29] R. J. Scherrer and M. S. Turner, Phys. Rev. D 33, 1585 (1986); 34, 3263(E) (1986).

[30] J. R. Ellis, D. V. Nanopoulos, L. Roszkowski, and D. N. Schramm, Phys. Lett. B 245, 251 (1990).

[31] K. Enqvist, K. Kainulainen, and J. Maalampi, Nucl. Phys. B317, 647 (1989).

[32] J. D. Vergados, C. C. Moustakidis, and V. Oikonomou, AIP Conf. Proc. 878, 138 (2006).

[33] V. K. Oikonomou, J.D. Vergados, and C.C. Moustakidis, Nucl. Phys. B773, 19 (2007).

[34] J. D. Lewin and P. F. Smith, Astropart. Phys. 6, 87 (1996).

[35] M. W. Goodman and E. Witten, Phys. Rev. D 31, 3059 (1985).

[36] D. S. Akerib et al. (CDMS Collaboration), Phys. Rev. D 73, 011102 (2006). 